\title{
Overview of Relay Selection and Dynamic Resource Allocation for Cooperative Wireless Network
}

\author{
Priyanka \\ ( A.P.) BIT, Meerut \\ U.P., India
}

\author{
Usha Pal \\ ( A.P.) BIT, Meerut \\ U.P., India
}

\author{
Neeraj Kumar \\ (A.P.) BIT, Meerut \\ U.P. India
}

\begin{abstract}
In the past decades, increasing demand for wireless multimedia and interactive Internet services, along with rapid proliferation of a multitude of communications and computational gadgets, are fuelling intensive research efforts on the design of novel wireless communication systems architectures for high speed, reliable and cost effective transmission solutions. Cooperative relay communication is a new paradigm shift for the next generation wireless system that will guarantee high data rates to all users in the network. It is anticipated to be the key technology aspect in the fifth generation wireless networks. In this Paper, we use system level simulations to gain insight into and quantify the influence of channel state information feedback delay in a multi-cell cooperative wireless system, and focus is put in relay selection and resource allocation for its optimal use. We analyzed the interplay between scheduling, power control in a multi-cell environment when the channel state information is subject to feedback delay. Detailed analysis is carried out using MATLAB and subsequent results are thoroughly discussed for deeper insight into the dynamics of cooperative networks.
\end{abstract}

\section{General Terms}

OFDMA, Cooperative relay selection, resource allocation, LTE.

\section{INTRODUCTION}

Orthogonal frequency division multiple access (OFDMA) based cooperative wireless network is a promising technology that is currently being standardized for various wireless access networks, notably for the long term evolution (LTE) of 3G networks [1]. In OFDMA networks, radio resource management (RRM) techniques are used to maximize system throughput as well as to provide appropriate quality of service (QoS) for end users.

To this end, dynamic packet assignment (scheduling), power control, and link adaptation (LA) and the interplay between these functions play an important role.

In broad terms, scheduling is responsible for assigning timefrequency resources to a certain (group of) users. Transmission power control is used to improve system throughput (e.g. water filling) and to maintain fairness by helping cell edge users reach a certain signal-to-interferenceand-noise (SINR) target. Link adaptation (LA) employs channel adaptive modulation and coding rate to increase the achievable data rate (spectrum efficiency) according to the channel conditions. In systems employing multiple antennas, antenna group selection and dynamic (multi-mode) precoding, including the adaptation of the transmission streams provide examples of adaptive resource management techniques [2].

In order for dynamic and adaptive RRM functions to work, obtaining channel state information (CSI) is crucial, because it is the basic input to these functions. Unfortunately, wireless channel characteristics vary rapidly in time - often at the time scale of milliseconds - which makes the application of adaptive radio resource management techniques non-trivial. In particular, wireless channel measurements typically suffer from delays and errors, both in the time and the frequency domains, which pose a challenge specifically on CSI estimation.

More specifically, precise and instantaneous CSI is not available due to:

1. Resource limitation: In order to save resources, only a limited time-frequency resources can be used for CSI measurements and reporting between mobile stations and radio base stations.

2. Measurement errors: equipment imprecision, quantization noise.

3. Feedback delay: the CSI estimation based on prior measurements can only be used for subsequent data transmissions. In practical systems, protocol aspects and resource limitations introduce a feedback delay that is typically a multiple of the time unit used for scheduling, power control and link adaptation decisions. The first aspect has been pointed out by [2] that provided an overview of recent works on limited feedback communications. The second problem is discussed in, for instance, and the references therein.

While all three aspects have been extensively studied in isolation, the joint system level impact of the above three factors, especially in multi-cell systems, is less understood and analyzed when employing realistic scheduler, power allocation and mobility models.

Therefore, the purpose of this thesis is to develop a system level model that is applicable to study the usefulness of link adaptation in a multi-cell OFDM system in the presence of CSI delay. Thereby, our purpose is to gain insight into the practical applicability and to verify the performance gains of dynamic adaptive modulation and coding schemes in realistic, multi-cell OFDM systems. While we take into account the first two aspects above, our focus is on the impact of the CSI feedback delay, because it appears to be particularly important and yet little understood area.

\section{SYSTEM MODEL}

In this study, we consider the uplink OFDM multi-user multicell cooperative system. And we confine our study within single input single output (SISO) scenario. In each cell, a number of users can transmit simultaneously. Due to the existence of interference, channel reciprocity cannot be reliably used regardless of duplex schemes. The base station is responsible for resource allocation. Firstly, it has to be informed of the user channel conditions. This could be done by detecting the sounding symbols or data transmitted from 
the intended user. After collecting all necessary channel state information (CSI) provided by users at time instant $t_{1}$, the base station makes scheduling, power control and link adaptation decisions which are fed back to users at time instant $t_{2}$. Subsequently, the scheduled users follow the resource allocation decisions and start to transmit at time instant $t_{3}$. The CSI inputs for RRM reflect the channel state at $t_{1}$, which however guides the user transmission format at $t_{3}$. For RRM to work efficiently there is an underlying assumption:

$\operatorname{CSI}\left(t_{3}\right) \approx C S I\left(t_{1}\right)$, or say, $C S I(t)=C S I(t-\tau)$, $\tau=t_{3}-t_{1}$

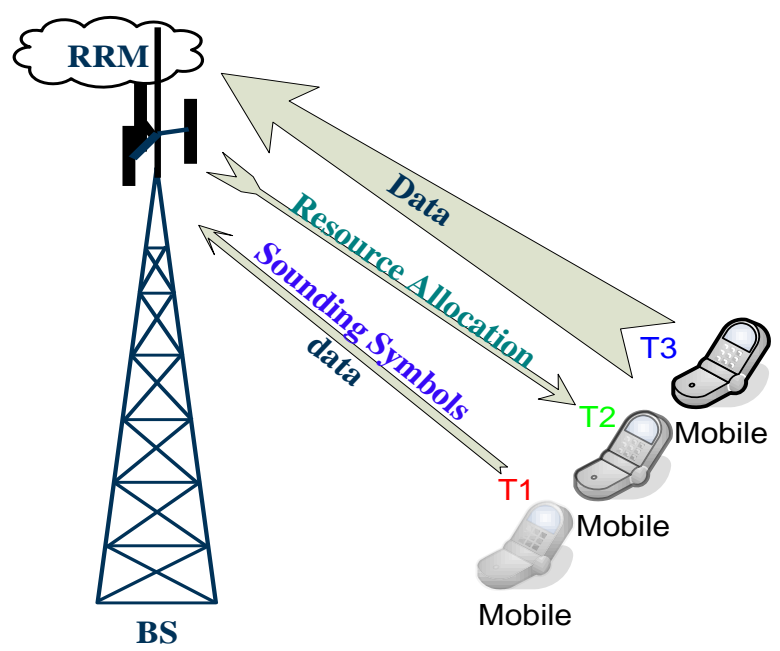

Fig. 1 Sketch map showing uplink CSI delay

The below flow chart shows that how RRM functions work in our system. The outdated CSI is the input to the scheduling, power control and link adaptation functions. After each transmission, we get an ideal CSI at the transmission moment. However, when this CSI is used to guide future transmission, it becomes outdated.

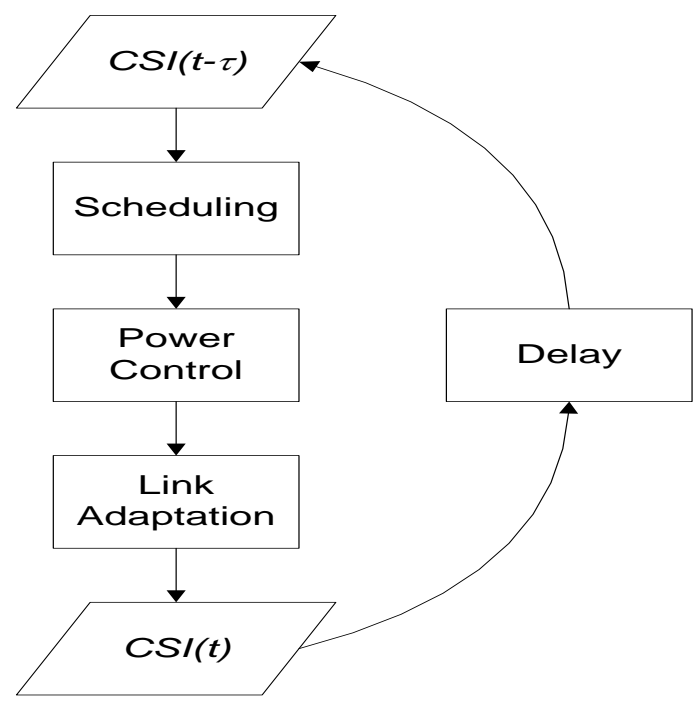

Fig. 2 Flow chart revealing relation between RRM functions and CSI while considering delay

\section{SIMULATION RESULT AND}

\section{ANALYSIS}

In this Section, we will test the performance of our proposed as well as reference schedulers in a multi-cell system. Feedback delay and CSI estimation error are considered for scheduling and link adaptation algorithms. The main criteria for evaluation are system and user throughput. Inter-cell interference and CSI variation level are also studied in different scheduling and user speed scenario. Basic assumptions are same as single-cell system.
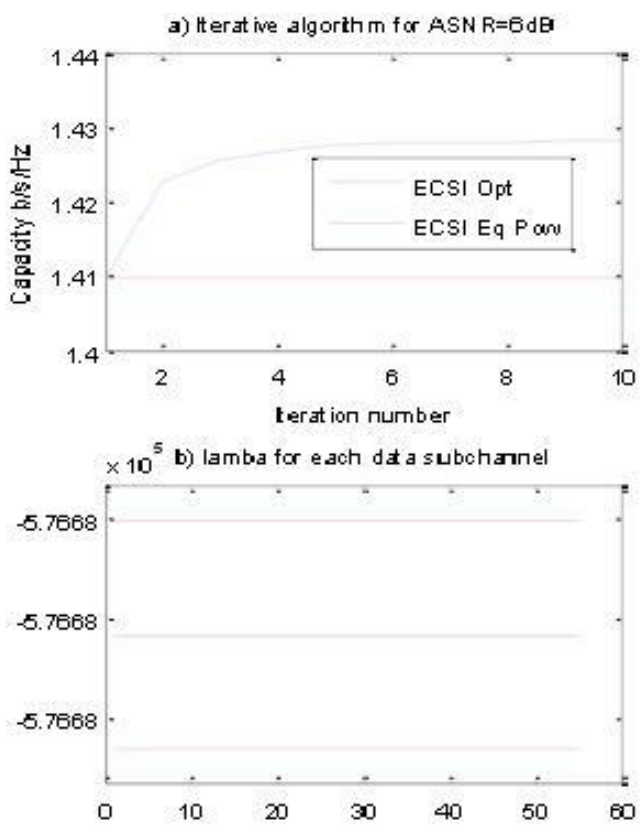

Figure.3 Performance of Iterative Algorithm

Figure 3 shows the performance of the proposed iterative algorithm. ASNR is $6 \mathrm{~dB}, \mathrm{~N}_{\mathrm{p}}=\mathrm{L}$. Figure 3.a. shows that within a few numbers of iterations the algorithm converges to the optimum point from the initial point, which is the equal power allocation. Figure 3.b. shows the computed lambda. As it is expected, the value of is the same for all data subchannel, which satisfies the analytical computation.
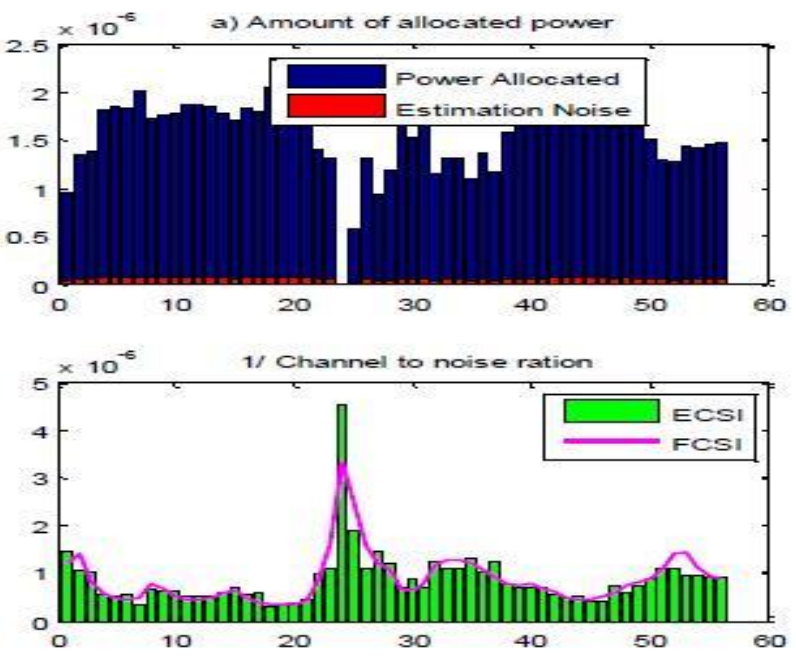

Figure 4 Distribution of Power with respect to Channel State Information 
Figure. 4 shows that the worse the estimated sub-channel response is, the less the amount of power allocated to that subchannel is. The algorithm performs like water-filling algorithm. Figure 5 is the same as figure 4, but the ASNR is $15 \mathrm{~dB}$. It can be seen that power allocation strategy has a tendency to allocated power among data subcarriers equally.
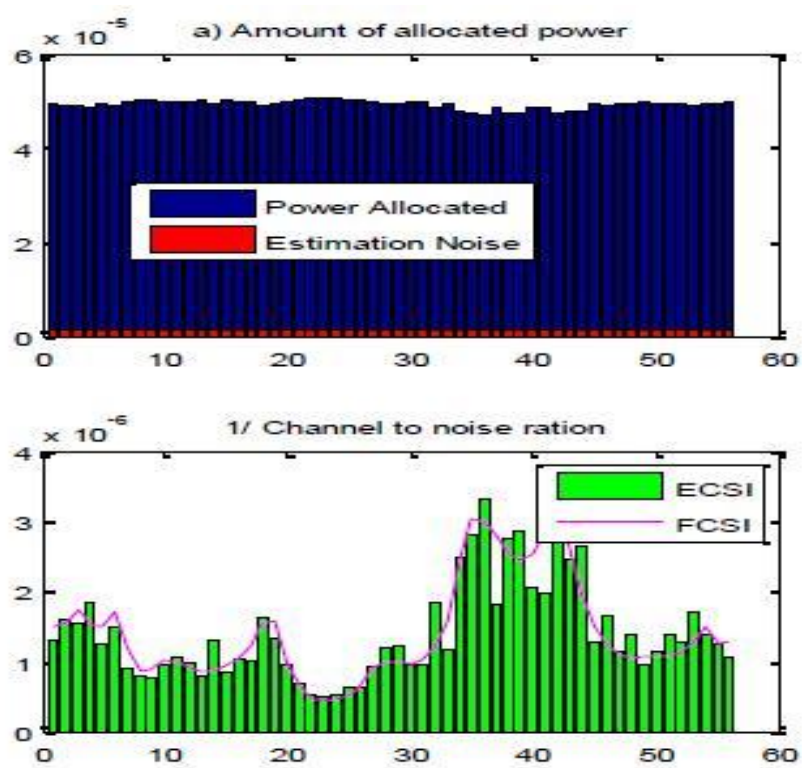

Figure 5 Distribution of Power with respect to Channel State Information

To further evaluate the iterative algorithm, other feasible initial points have been chosen and all converge to the optimum solution but with different number of iteration. Among all, equal power distribution generally has a lowest number of iteration to converge. For figures 6,7 , and 8 , the iterative algorithm is used to find the optimum result. The value of lambda for each data subcarriers is computed and if these values were in the region on uncertainty we end the iteration.

In figure 6, the amount of ASNR is sweeped and the system performance is depicted. In this figure alpha is fixed. It is obvious from figure that equal power distribution among all subcarriers is near optimum for ASNR greater than $6 \mathrm{~dB}$. The dashed line is depicted to show the $3 \mathrm{~dB}$ performance degradation in system capacity as described in. The computation shows that in high SNR for the, the performance relative to full channel state information degrade about $3 \mathrm{~dB}$ and one can see the issue in this figure.

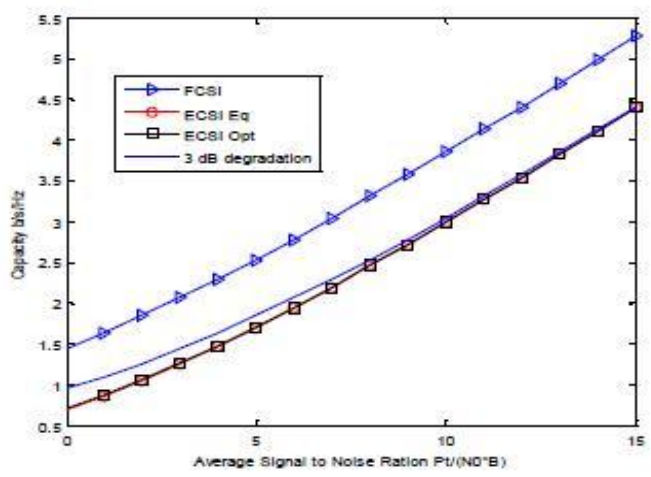

Figure .6 System Performances versus ASNR
In figure 7 , fixed value of $\mathrm{ASNR}=15 \mathrm{~dB}$, the system performance versus different values of alpha is illustrated. It can be seen that there is an optimum value for parameter alpha, in which the graph peaks. The green dashed lines are the optimum value of alpha computed. Since the ASNR is considered almost high in this simulation, the equation is approximately the optimum one as it is indicated in figure 8 . In figure 8 , the deviation of alpha, from the equation, for two values of ASNR is depicted.

As stated before, for high ASNR, the alpha converges to optimal value which is also shown in the figure. Moreover, there is little difference between uniform channel power delay profile and uniform ones. However, the difference becomes larger as the ASNR decreases.

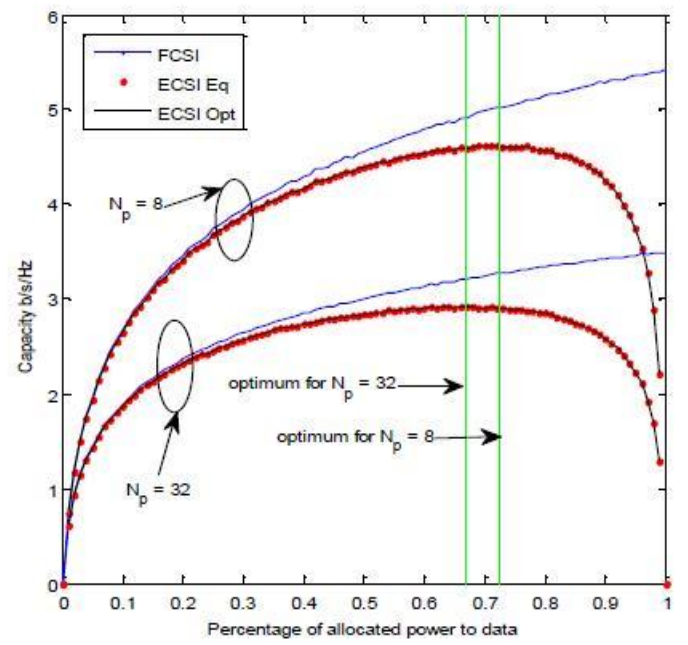

Figure.7 System performance versus ratio of allocation power

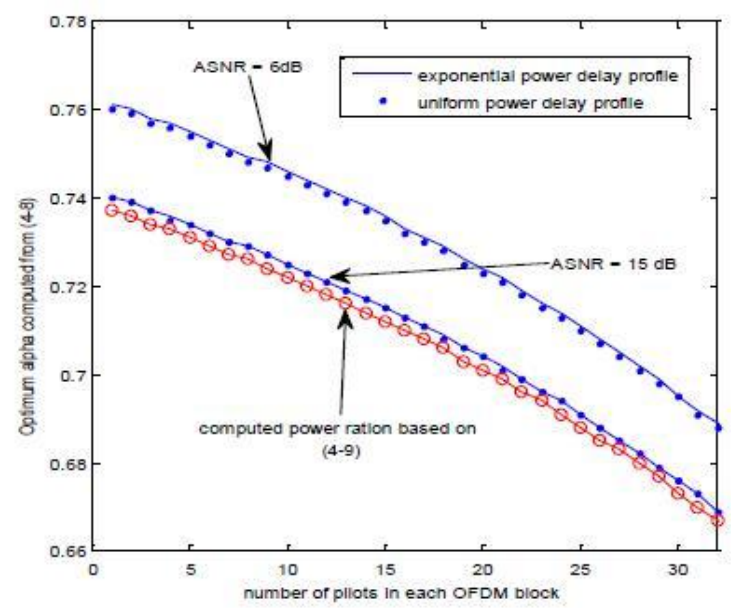

Figure .8 Theoretical and calculated value of optimal alpha

The performance of the system with our approach can be judge by the BER and Eb/No graphs. Figure 9 shows the graph comparison and we can see our graph in fig. 9 shows better performance as compare to old one [4] because it gives the straighter curve. 


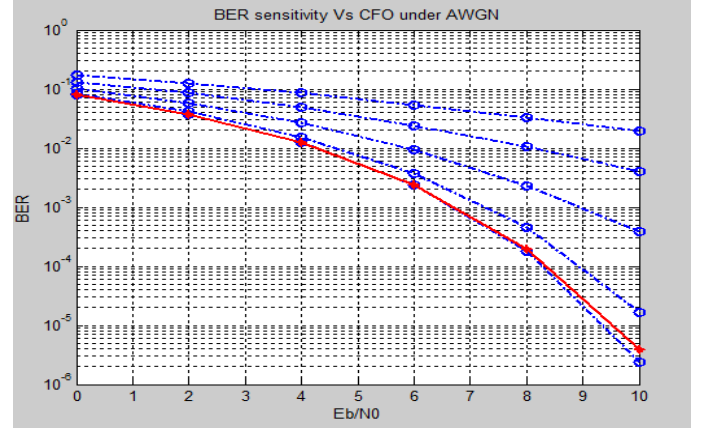

Figure.9 Performance graph between BER AND $\mathrm{E}_{\mathrm{b} /} \mathbf{N}_{\mathbf{0}}$

\section{CONCLUSION}

In this Paper, we studied how power control strategies and different types of scheduling can affect the performance of the system like throughput and coverage in uplink OFDM based cooperative system. We employed system model and simulation tools which can account for channel state information (CSI) feedback delay and link adaptation errors. We also carried out detailed simulation of power allocation and relay selection strategies with different CSI parameters and find out that through our schemes we can make every over-all system throughput to significantly improve through channel capacity maximization.

\section{FUTURE WORKS}

Radio resource management is responsible for adapting transmission parameters to ever changing channel conditions in wireless communication systems. For radio management functions to work there's a presumption that we could obtain channel state information accurately. However, due to some factors such as feedback delay, the channel state information can never be ideal. To combat this delay problem and approach as accurate CSI value as possible, there are mainly two ways to get there

\section{Smooth CSI variation in time.}

In this case, CSI value obtained from previous measurement is still valid for future transmissions. That is, make the delayed channel state information as valuable as instantaneous one. This is what basically we do in the thesis. But of course, there is another alternative to cope with the feedback delay of CSI.

2. Advanced prediction algorithm

If we can predict future CSI based on previous measurement, we don't have to worry about the CSI variation in that case. This is a very interesting area to be exploited in the future. Furthermore, resource allocation strategy using different optimization techniques can also be carried out in immediate future as a natural extension of this work

\section{REFERENCES}

[1] 3GPP TR 25.814 V7.1.0,"Physical Layer Aspects for Evolved Universal Terrestrial Radio Access (E-UTRA)", Release 7, September 2006.

[2] Catreux, S.; Erceg, V.; Gesbert, D.; Heath, R.W., Jr.; "Adaptive modulation and MIMO coding for broadband wireless data networks", Communications Magazine, IEEE Volume 40, Issue 6, June 2002 Page(s):108 - 115

[3] 3GPP TS 36.213 V8.0.0, "Physical Layer Procedures", Release 8, September 2007.

[4] Chi-Min Li : Performance Comparison of the STBCOFDMA Decoders in a Fast Fading Channel, Journal of Marine Science and Technology, Vol. 20, No. 5, pp. 534540 (2012)

[5] Bonnet, J.; Auer, G.; "Chunk-based Channel Estimation for Uplink OFDM", Vehicular Technology Conference, 2006. VTC 2006-Spring. IEEE 63rd Volume 4, 2006 Page(s):1555 - 1559

[6] Valenti, M.C.; Woerner, B.D.;'Iterative channel estimation and decoding of pilot symbol assisted turbo codes over flat-fading channels", Selected Areas in Communications, IEEE Journal on Volume 19, Issue 9, Sept. 2001 Page(s):1697 - 1705

[7] 3GPP "3rd generation partnership project; Technical specification group radio access network; Spatial channel model for Multiple Input Multiple Output (MIMO) simulations", 3GPP TR 25.996, V6.1.0

[8] Love, D.J.; Heath, R.W., Jr.;"Multimode preceding for MIMO wireless systems" Signal Processing, IEEE Transactions on [see also Acoustics, Speech, and Signal Processing, IEEE Transactions on] Volume 53, Issue 10, Part 1, Oct. 2005 Page(s):3674 - 3687

[9] Gabor Fodor and Chrysostomos Koutsimanis"A Low Intercell Interference Variation Scheduler for OFDMA Networks", IEEE International Conference on Communications (ICC '08), Beijing, China, May 2008.

[10] Hongwei Yang;"A road to future broadband wireless access: MIMO-OFDM-Based air interface", Communications Magazine, IEEE Volume 43, Issue 1, Jan. 2005 Page(s):53 - 60 